\title{
Stereotactic radiosurgery for melanoma brain metastases: dose-size response relationship in the era of immunotherapy
}

\author{
Mehran Yusuf $^{1} \cdot$ Abbas Rattani $^{2}$ (D) Jeremy Gaskins ${ }^{3} \cdot$ Alexandria L. Oliver $^{4} \cdot$ Steven F. Mandish $^{2} \cdot$ Eric Burton $^{5}$. \\ Michael E. May ${ }^{2} \cdot$ Brian Williams $^{6} \cdot$ Dale Ding $^{6} \cdot$ Mayur Sharma $^{6} \cdot$ Donald Miller $^{7} \cdot$ Shiao Woo $^{2}$
}

Received: 23 August 2021 / Accepted: 11 November 2021 / Published online: 22 November 2021

(c) The Author(s), under exclusive licence to Springer Science+Business Media, LLC, part of Springer Nature 2021

\begin{abstract}
Purpose/Objective(s) To determine, for intact melanoma brain metastases (MBM) treated with single-fraction stereotactic radiosurgery (SRS), whether planning parameter peripheral dose per lesion diameter (PDLDm, Gy/mm) and lesion control (LC) differs with versus without immunotherapy (IO).

Materials/Methods We performed a retrospective analysis of patients with intact MBM treated with SRS from 2008 to 2019. Cox-frailty models were constructed to include confounders selected by penalized Cox regression models with a LASSO selector. Interaction effect testing was used to determine whether a significant effect between IO and PDLDm could be demonstrated with respect to LC.

Results The study cohort comprised 67 patients with 244 MBMs treated with SRS (30 patients with 122 lesions treated with both SRS and IO) were included. The logarithm of PDLDm was selected as a predictor of LC (HR 0.307, 95\% CI 0.098-0.441), adjusting for IO receipt (HR 0.363, 95\% CI 0.108-1.224). Interaction effect testing demonstrated a differential effect of PDLDm by IO receipt, with respect to LC $(\mathrm{p}=0.048)$. Twelve-month LC rates for a $7.5 \mathrm{~mm}$ lesion receiving SRS (18 Gy) with IO versus without IO were 87.8\% (95\% CI 69.0-98.3\%) versus 79.8\% (95\% CI 55.1-93.8\%) respectively. Conclusion PDLDm predicted LC in patients with small MBMs treated with single-fraction SRS. We found a differential effect of dose per lesion size and LC by immunotherapy receipt. Future studies are needed to determine whether lower doses of single-fraction SRS afford similarly effective LC for patients with small MBMs receiving immunotherapy.
\end{abstract}

Keywords Stereotactic radiosurgery $\cdot$ Immunotherapy $\cdot$ Melanoma $\cdot$ Brain metastasis $\cdot$ Dose $\cdot$ Size

\section{Abbreviations}

BM Brain Metastasis

CI Confidence Interval

CNS Central Nervous System

DBF Distant Brain Failure

Mehran Yusuf and Abbas Rattani are the co-first authors.

Abbas Rattani

abbas.rattani@louisville.edu

Jeremy Gaskins

jeremy.gaskins@louisville.edu

1 Department of Radiation Oncology, University of Alabama at Birmingham, Birmingham, AL, USA

2 Department of Radiation Oncology, School of Medicine, University of Louisville Hospital, 529 S. Jackson St, Louisville, KY 40202, USA

3 Department of Bioinformatics and Biostatistics, University of Louisville, Louisville, KY, USA
HR Hazard Ratio

IO Immunotherapy

LASSO Least Absolute Shrinkage and Selection Operator

LC Local Control

LF Local Failure

MBM Melanoma Brain Metastases

4 School of Medicine, University of Louisville, Louisville, KY, USA

5 Neuro-Oncology Branch, National Cancer Institute, National Institutes of Health, Bethesda, MD, USA

6 Department of Neurosurgery, University of Louisville Hospital, Louisville, KY, USA

7 Department of Medical Oncology, University of Louisville Hospital, Louisville, KY, USA 


$\begin{array}{ll}\text { MR } & \text { Magnetic Resonance } \\ \text { OS } & \text { Overall Survival } \\ \text { PDLDm } & \text { Peripheral Dose Per Lesion Diameter } \\ \text { PTV } & \text { Planning Target Volume } \\ \text { Q2 Weekly } & \text { Every Second Week } \\ \text { Q3 Weekly } & \text { Every Third Week } \\ \text { RANO } & \text { Response Assessment in Neuro-Oncology } \\ \text { RN } & \text { Radiation Necrosis } \\ \text { RPA } & \text { Recursive Partitioning Analysis } \\ \text { RTOG } & \text { Radiation Therapy Oncology Group } \\ \text { SRS } & \text { Stereotactic Radiosurgery } \\ \text { V12 } & \text { Brain volume receiving 12 Gray } \\ \text { WBRT } & \text { Whole Brain Radiotherapy }\end{array}$

\section{Introduction}

Melanoma patients have a high incidence of both synchronous and metachronous de novo brain metastases [1]. Stereotactic radiosurgery (SRS) has been demonstrated to achieve high rates of lesion control in multiple published series for appropriately selected melanoma brain metastases (MBM) [2-4]. Achieving intracranial disease control has become increasingly important, given the paradigm-shifting improvement in clinical outcomes for selected patients with locally advanced or metastatic melanoma with the advent of novel immune-modulating therapies. Although multiple published series have suggested improved lesion control with combined modality therapy (SRS and immunotherapy), some studies have reported higher rates of radiation necrosis (RN) [5,6]. Given that the SRS dose levels specified by the Radiation Therapy Oncology Group (RTOG) trial 90-05 [7] were defined by maximal tolerated dose, there has been interest in determining SRS doses associated instead with optimal lesion control while simultaneously limiting normal brain toxicity. Subsequent retrospective studies have suggested there is a dose-volume (size) response relationship for intact brain metastases receiving single-fraction SRS associated with lesion control and that peripheral dose per lesion diameter (PDLDm, Gy/mm) may be a useful SRS planning parameter $[8,9]$.

We performed a retrospective analysis of a prospectively maintained cohort of consecutive intact MBM patients treated with single-fraction SRS to determine whether the relationship between the SRS planning parameter PDLDm and lesion control differed with versus without immunotherapy (IO). If small MBMs treated with SRS and IO do not demonstrate the same benefit from higher PDLDm with regards to lesion control as MBMs treated with SRS alone, one may question whether prescribed doses $>18$ Gy are necessary to obtain high rates of lesion control for small MBMs treated with SRS and IO. This finding would carry clinical significance, providing further rationale for studies investigating SRS dose de-escalation in the setting of concomitant IO administration [10]. The overall aim is to maintain excellent rates of lesion control with potentially decreased risk of radiation necrosis.

\section{Materials and methods}

\section{Patient cohort}

We performed a retrospective analysis at a single institution from 2008 (following institution transition to ARIA®) treatment planning system) to 2019 (prior to the COVID19 pandemic) of MBM patients treated with SRS who either received or did not receive IO. This study aimed to assess whether the exposure of immunotherapy in MBM patients receiving SRS impacted the SRS planning parameter PDLDm and whether lesion control differed between cohorts. Institutionally, we avoid whole brain radiation (WBRT) for patients with melanoma brain metastases in favor of single or fractionated stereotactic radiation therapy if feasible. The very small number of patients who receive WBRT with melanoma brain metastases at our institution are typically patients with innumerable brain metastases, poor Karnofsky Performance Scale score, and concordantly poor prognosis limiting ability to meaningfully characterize treatment effects or clinical outcomes. Institutional practice was to fractionate brain metastasis $\geq 20 \mathrm{~mm}$; therefore, small brain metastasis (BM) was defined as $<20 \mathrm{~mm}$. IO was administered either concurrently or within 12 weeks of receiving SRS, and it included anti-PD-1, anti-CTLA-4, and combination anti-PD-1/anti-CTLA-4 therapy. Standard dosing was $3 \mathrm{mg} / \mathrm{kg}$ every third week (Q3 weekly) for up to 4 cycles for anti-CTLA-4 therapy, $2 \mathrm{mg} / \mathrm{kg}$ or $200 \mathrm{mg}$ IV Q3 weekly until disease progression or unacceptable toxicity for anti-PD1 therapy with pembrolizumab, and $240 \mathrm{mg}$ IV every second week (Q2 weekly) until disease progression or unacceptable toxicity for anti-PD1 therapy with nivolumab. Patients receiving combination received ipilumumab ( $3 \mathrm{mg} /$ $\mathrm{kg}$ ) and nivolumab (1 mg/kg) IV Q3 weekly for up to 4 cycles with maintenance nivolumab (240 mg IV Q2 weekly) until disease progression or unacceptable toxicity.

\section{SRS treatment technique}

Patients were included if they were treated using one of two relevant machines available at our institution: the Varian Trilogy Linear Accelerator (Varian, Palo Alto, California, USA) or the CyberKnife linear accelerator (Accuray, Sunnyvale, California, USA). All patients were immobilized with the use of a thermoplastic face mask in the supine position in accordance with standard practice. MRI of the whole brain with $0.75 \mathrm{~mm}$ thin slices was fused to the treatment planning 
simulation CT for optimal target delineation. The planning target volume was defined as the contrast enhancing lesion delineated on $\mathrm{T} 1$ weighted imaging sequences with gadolinium, incorporating both targeting and setup error. An additional planning target volume (PTV) margin was not routinely added at our center to minimize potential toxicity from increased dose to adjacent normal brain parenchyma [11].

\section{Failure criteria}

Local failure was defined using a modified evaluation criteria in solid tumors (RECISTV1.1). All patients in this cohort received one SRS treatment. Therefore, MBM requiring surgical intervention with pathologic confirmation of viable malignancy, requiring additional SRS, or progressive growth (defined as $>20 \%$ increase in diameter with a minimum increase of $5 \mathrm{~mm}$ ) on MR imaging were considered local failures. Further, an additional criterion of serial growth on 2 consecutive MR imaging sequences was used.

Distant brain failure was defined by the development of additional (new) brain metastases after radiosurgery determined by imaging, additional intracranial radiation (SRS or whole brain radiotherapy (WBRT)), or surgical resection of a non-previously known brain metastasis. Overall survival (OS) was defined as time from SRS to death from any cause. Radiation necrosis was defined/determined by consensus opinion from our institutional multidisciplinary neurooncology tumor board comprised of radiation oncologists, neuro-oncologists, neurosurgeons, and neuroradiologists as recommended by guidelines from the RANO group [12]. Advanced imaging sequences including MR spectroscopy, MR perfusion, T2 weighted sequences to assess edema, and diffusion-weighted imaging were used and assessed by the multidisciplinary tumor board to help differentiate RN from recurrent or residual disease. Symptomatic radiation necrosis was defined as RN meeting the aforementioned criteria requiring intervention such as steroids, bevacizumab, or craniotomy. Our institutional practice, which informed our protocol has been to obtain an MRI one month after treatment completion, every 3 months within the first year in absence of new neurologic symptoms, every 4 months in the second year with consideration of further spacing pending neurologic symptoms and development of intracranial/ extracranial disease progression.

\section{Statistical analyses}

All analyses were performed using the R statistical software (R Foundation for Statistical Computing, Vienna, Austria). Baseline patient demographic, lesion and treatment factors were collected and adjusted for in the performed analyses. Descriptive statistics were used to describe both the overall patient and lesion cohort subgroups stratified by IO receipt. Clinical outcomes of interest included freedom from local failure (LF), freedom from distant brain failure (DBF), overall survival and symptomatic radiation necrosis.

Kaplan-Meier methods were used to estimate survival outcomes including freedom from LF with a frailty term [13] to account for multiple lesions within the same patient. Cox models were fit to assess the impact of IO on each of the clinical outcomes with a frailty term included for outcomes measured on the lesion-level. Multivariable Cox frailty models were constructed to include confounders selected by penalized Cox regression models with a least absolute shrinkage and selection operator (LASSO) selector with IO always included as the main predictor of interest. Interaction effect testing was used to determine whether IO modulated the relationship between LF and PDLDm in the Cox frailty model. To reduce skewness, a log-transformation of PDLDm is used in all Cox models, but results are reported back on the corresponding (non-logarithmic) scale. The probability of freedom from LF at 12 months was estimated from the Cox frailty model with interaction, and curves depicting the relationship between these predicted probabilities with and without IO across the range of PDLDm values are reported.

The choice of parametric vs non-parametric test was based on sample distribution. When data were approximately bell-shaped, parametric test (two-sample t-test) was used. When certain characteristics displayed substantially non-normality — such as small counts (e.g. number of brain metastases), skewed variables (e.g. tumor size characteristics), and variables with substantial ties/repeated values (e.g. peripheral dose)—for such variables and the moderate cohort size, the non-parametric test (Mann-Whitney U test) was used and summarized as median and inter quartile range (IQR). To denote which variables are summarized using non-parametric methods, we displayed the IQR in brackets to distinguish from the sample standard deviation for the approximately normal variables (shown as a single number).

\section{Results}

\section{Patient characteristics}

A summary of patient and tumor characteristics, as well as treatment factors are outlined in Table 1. The study cohort was comprised of 67 consecutive patients with an average of 61 years of age and a total of 244 MBMs. The median follow-up time for patients receiving SRS alone was 6.9 months and 8.4 months for patients receiving SRS with IO $(p=0.284)$. A significant difference was appreciated in the year of diagnosis, wherein the SRS alone cohort were diagnosed in 2010 and the SRS with immunotherapy were diagnosed in $2015(\mathrm{p}<0.001)$. Of the 67 included patients, 
Table 1 Baseline Patient Level Demographic, Tumor and Treatment Factors

\begin{tabular}{|c|c|c|c|c|c|c|}
\hline & & \multicolumn{2}{|c|}{ SRS Alone } & \multicolumn{2}{|c|}{$\begin{array}{l}\text { SRS with Immuno- } \\
\text { therapy }\end{array}$} & \multirow[t]{2}{*}{ P-value } \\
\hline & & $\mathrm{N}=37$ & $55 \%$ & $\mathrm{~N}=30$ & $45 \%$ & \\
\hline Female Biologic Sex & & 12 & $32 \%$ & 9 & $30 \%$ & 1.000 \\
\hline Age & & 61.8 & 14.4 & 61.1 & 13.0 & 0.825 \\
\hline \multirow[t]{6}{*}{ KPS } & & & & & & 0.070 \\
\hline & 60 & 2 & $5 \%$ & 0 & $0 \%$ & \\
\hline & 70 & 6 & $16 \%$ & 1 & $3 \%$ & \\
\hline & 80 & 8 & $22 \%$ & 8 & $27 \%$ & \\
\hline & 90 & 18 & $49 \%$ & 15 & $50 \%$ & \\
\hline & 100 & 3 & $8 \%$ & 6 & $20 \%$ & \\
\hline Brain Metastasis at DX & & 2 & {$[1-3]$} & 2 & {$[2-4]$} & 0.251 \\
\hline At least one Body Metastasis at DX & & 29 & $78 \%$ & 23 & $77 \%$ & 1.000 \\
\hline \multirow[t]{4}{*}{ RPA } & & & & & & 0.295 \\
\hline & 1 & 5 & $14 \%$ & 3 & $10 \%$ & \\
\hline & 2 & 29 & $78 \%$ & 27 & $90 \%$ & \\
\hline & 3 & 3 & $8 \%$ & 0 & $0 \%$ & \\
\hline \multirow[t]{4}{*}{ BRAF gene mutation } & & & & & & 0.289 \\
\hline & Negative & 6 & $38 \%$ & 10 & $63 \%$ & \\
\hline & Positive & 10 & $63 \%$ & 6 & $38 \%$ & \\
\hline & Unknown & 21 & & 14 & & \\
\hline Prior Chemotherapy & & 24 & $65 \%$ & 8 & $26 \%$ & 0.003 \\
\hline Prior Immunotherapy & & 3 & $8 \%$ & 4 & $13 \%$ & 0.692 \\
\hline Post Immunotherapy & & 4 & $11 \%$ & 5 & $17 \%$ & 0.500 \\
\hline Concurrent SRS & & & & 27 & $90 \%$ & \\
\hline Extracranial Control at SRS & & 8 & $22 \%$ & 7 & $23 \%$ & 1.000 \\
\hline Length of Follow-up (mo) & & 6.9 & {$[3.0-11.6]$} & 8.4 & {$[3.7-22.3]$} & 0.284 \\
\hline Diagnosis Year & & 2010 & ['10-'13] & 2015 & ['13-'17] & $<.001$ \\
\hline
\end{tabular}

Continuous variables reported with mean, standard deviation, and t-test p-value; unordered categorical variables reported as n, sample proportion, and Fisher test p-value; ordered categorical variables (KPS and RPA) reported as $\mathrm{n}$, sample proportion, and Mann-Whitney test $\mathrm{p}$-value; non-parametric variables reported as median, [IQR in brackets], and Mann-Whitney test p-value

$D X$ diagnosis, $I Q R$ interquartile range, KPS Karnofsky Performance Scale, mo month(s), RPA recursive partitioning analysis, $S R S$ stereotactic radiosurgery
55\% $(\mathrm{n}=37)$ received SRS alone. Approximately half of patients had a Karnofsky Performance Scale score of $90 \%$. Nearly $78 \%$ of patients also had at least 1 body metastasis at time of diagnosis. In patients receiving SRS alone (and known BRAF status), $63 \%$ were BRAF positive (compared to $38 \%$ who also received IO), and $63 \%$ were BRAF negative in those receiving SRS and IO (compared to 38\% in SRS alone). No significant difference was present with regards to BRAF mutational status $(\mathrm{p}=0.289)$. In the SRS alone cohort, $65 \%$ of patients had received prior chemotherapy compared to $26 \%$ in the SRS and IO group ( $p=0.003)$.

Seventy-five lesions were treated using the Varian Trilogy Linear Accelerator and 169 lesions were treated using the CyberKnife linear accelerator. Lesion characteristics and treatment factors are summarized in Table 2. A total of 244 lesions were captured across 67 patients in this study. Thirty patients or 45\% (all receiving SRS and IO) represented a total of 122 lesions. Forty-one lesions were treated with antiPD-1 (36 and 5 lesions were treated with pembrolizumab and nivolumab respectively), 52 lesions were treated with anti-CTLA-4, and 29 lesions were treated with combination ipilumumab and nivolumab with maintenance nivolumab until disease progression or unacceptable toxicity. Of the 122 lesions treated with SRS and IO, 61\% $(n=75)$ received SRS concurrently with IO (SRS administration within 28 days of IO administration), 14 lesions (11\%) were treated with SRS more than 28 days before IO administration, and 33 lesions (27\%) were treated with SRS more than 28 days after IO administration. Lesions treated with SRS alone had significantly higher rates of prior chemotherapy compared to those treated with SRS and IO (68\% vs $34 \%, \mathrm{p}<0.001)$. The median lesion diameter was similar for the SRS with IO (7.9 mm, IQR: $5.0-12.8 \mathrm{~mm}$ ) versus without IO (7.4 mm, IQR: $5.9-9.5 \mathrm{~mm})$ subgroups $(\mathrm{p}=0.811)$. The median 
Table 2 Baseline Lesion (Brain Metastasis) Level Tumor and Treatment Factors

\begin{tabular}{|c|c|c|c|c|c|c|}
\hline & & \multicolumn{2}{|c|}{ SRS Alone } & \multicolumn{2}{|c|}{$\begin{array}{l}\text { SRS with Immuno- } \\
\text { therapy }\end{array}$} & \multirow[t]{2}{*}{ P-value } \\
\hline \multicolumn{2}{|l|}{ n (lesions) } & 122 & $50 \%$ & 122 & $50 \%$ & \\
\hline \multicolumn{2}{|l|}{ number of patients* } & 40 & $60 \%$ & 30 & $45 \%$ & \\
\hline \multicolumn{7}{|l|}{ Immune Type } \\
\hline & Pembro or Nivo & & & 41 & $34 \%$ & \\
\hline & Ipi & & & 52 & $43 \%$ & \\
\hline & Ipi/Nivo & & & 29 & $24 \%$ & \\
\hline Diameter (mm) & & 7.4 & {$[5.0-9.5]$} & 7.9 & {$[5.0-12.8]$} & 0.811 \\
\hline Volume $\left(\mathrm{mm}^{3}\right)$ & & 147 & {$[64-460]$} & 160 & [52-659] & 0.840 \\
\hline Concurrent SRS & & & & 108 & $89 \%$ & \\
\hline Prior Chemotherapy & & 82 & $68 \%$ & 41 & $34 \%$ & $<.001$ \\
\hline Prior Immunotherapy & & 12 & $10 \%$ & 9 & $7 \%$ & 0.649 \\
\hline \multirow[t]{4}{*}{ BRAF Gene Mutation } & & & & & & $<.001$ \\
\hline & Negative & 16 & $28 \%$ & 32 & $70 \%$ & \\
\hline & Positive & 41 & $72 \%$ & 14 & $30 \%$ & \\
\hline & Unknown & 65 & & 76 & & \\
\hline \multicolumn{2}{|l|}{ Peripheral Dose (Gy) } & 18 & [18-18] & 18 & [18-18] & 0.486 \\
\hline \multicolumn{2}{|l|}{ Maximum Dose (cGy) } & 2250 & [2117-2352] & 2212 & [2152-2450] & 0.825 \\
\hline \multicolumn{2}{|l|}{ Mean Dose $(\mathrm{cGy})^{* *}$} & 2029 & [1955-2117] & 2008 & [1969-2119] & 0.974 \\
\hline \multicolumn{2}{|c|}{ Peripheral Dose (Gy) / Diameter (mm) } & 2.53 & {$[1.5-3.60]$} & 2.3 & {$[1.46-3.58]$} & 0.807 \\
\hline \multicolumn{7}{|l|}{ Treatment Schedule } \\
\hline & Concurrent & & & 75 & $61 \%$ & \\
\hline & SRS before IO & & & 14 & $11 \%$ & \\
\hline & IO before SRS & & & 33 & $27 \%$ & \\
\hline
\end{tabular}

Continuous variables reported with mean, standard deviation, and t-test p-value; unordered categorical variables reported as n, sample proportion, and Fisher test p-value; ordered categorical variables (KPS and RPA) reported as $\mathrm{n}$, sample proportion, and Mann-Whitney test $\mathrm{p}$-value; non-parametric variables reported as median, [IQR in brackets], and Mann-Whitney test p-value

$I O$ immunotherapy, Ipi ipilimumab, IQR interquartile range, Nivo nivolumab, Pembro pembrolizumab, SRS stereotactic radiosurgery

*Three patients had lesions that we treated using SRS alone as well as other lesions treated with SRS + IO

**77 lesion have unrecorded mean dose. For these lesions, values are imputed using the recorded maximum dose peripheral SRS dose was 18 Gy for both lesions treated with and without IO $(\mathrm{p}=0.486)$.

\section{Survival, failure, and side effect profile}

The 12-month OS rate was significantly higher for patients with at least one lesion treated with SRS and IO (48.5\%, CI 32-73.6\%) compared to patients treated with SRS alone (24.3\%, CI 13.8-42.9, p=0.015, Fig. 1a). The 12-month freedom from DBF rate was similar for patients treated with SRS and IO (30.4\%, CI 15.2-60.7\%) compared to patients receiving SRS alone $(20.0 \%$, CI $8.6-46.7 \%, \mathrm{p}=0.445$, Fig. 1b). With regards to lesion control, the 12-month freedom from local failure rate was also significantly higher for lesions treated with SRS and IO (83.9\%, CI 64.4-97.1\%) with respect to lesions treated with SRS alone $(75.3 \%$, CI $59.1 \%-87.8 \%, p=0.040$, Fig. 1c). The 12-month rate for freedom from symptomatic radiation necrosis was higher for patients receiving SRS with IO (100\%, CI 100-100\%) compared to patients receiving SRS alone (89\%, CI 76.4-97.4\%, $\mathrm{p}=0.049$, Fig. 1d). No significant difference in rates of 12-month freedom from asymptomatic radiation necrosis was present for lesions treated with SRS and IO (97.5\%, CI 93.5-100\%) versus lesions treated with SRS alone $(94.7 \%$, CI $87.8-99.1 \%)$ in the study cohort $(p=0.26)$. No significant difference with regards to freedom from LF was present in a univariable subgroup analysis of lesions treated with SRS and IO stratified by treatment sequence (concurrent, IO before SRS, SRS before IO, $\mathrm{p}=0.86$ ).

Cox frailty modeling with the endpoint of OS found IO receipt (Hazard Ratio (HR) 0.482, CI 0.265-0.879) to be a significant predictor of OS in the study cohort, and the potential confounders of biologic sex, Karnofsky Performance Scale, age, number of brain metastases at diagnosis, 


\section{(a) Overall Survival (by Patient)}

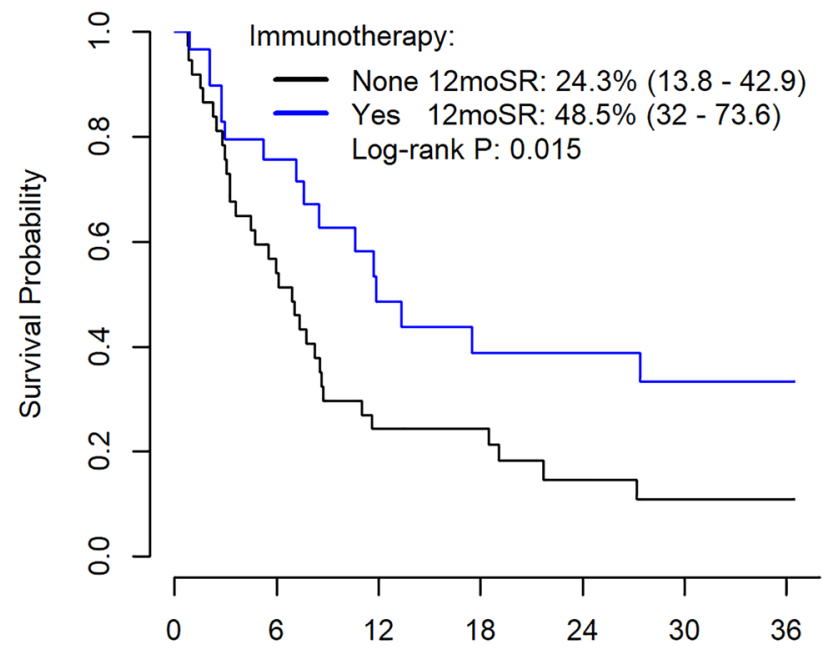

Months from completion of radiosurgery (c) Freedom from Local Failure
(by Lesion)

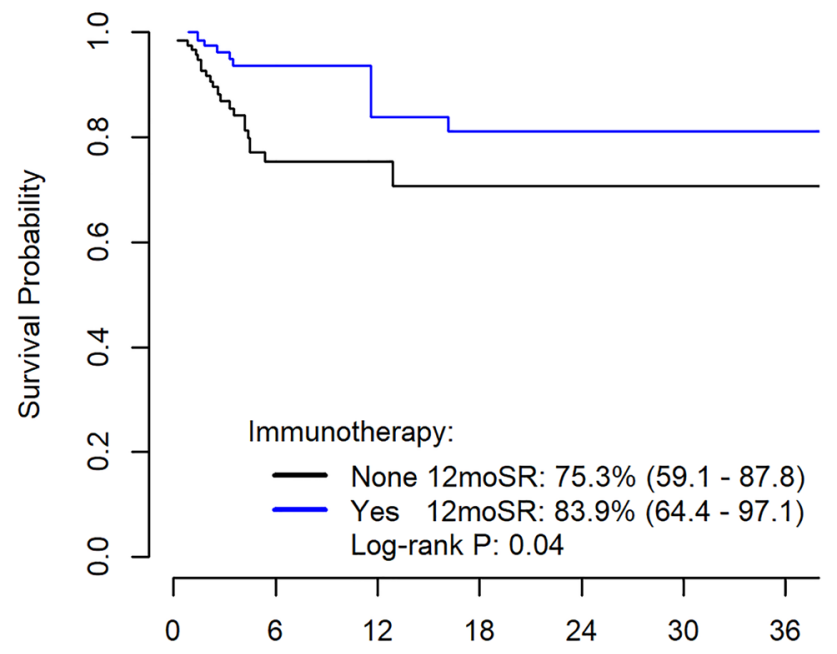

Months from completion of radiosurgery

Fig. 1 a Kaplan-Meier curve comparing OS for subgroups stratified by IO receipt. b Kaplan-Meier curve comparing freedom from DBF for subgroups stratified by IO receipt. c Kaplan-Meier curve

extracranial disease control time at diagnosis, extracranial disease control at time of SRS surgical resection, recursive partitioning analysis (RPA) class at diagnosis, BRAF mutational status, prior chemotherapy, and prior immunotherapy were all excluded by the LASSO selector (Table 3). Multivariable Cox frailty with the endpoint of DBF excluded all available factors (IO receipt HR 0.776, CI 0.405-1.489). With regards to lesion control, the SRS planning parameter (b) Freedom from Distant Brain Failure (by Patient)

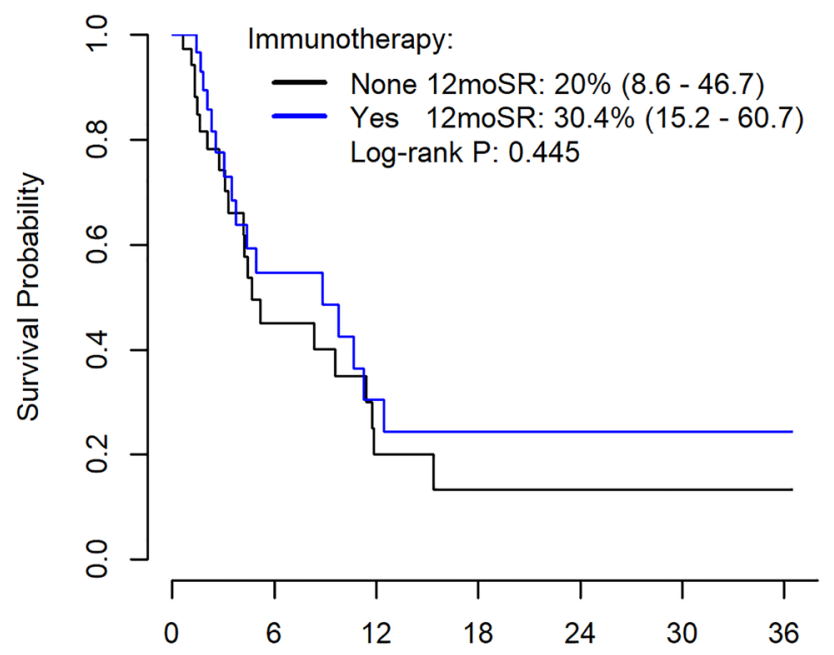

Months from completion of radiosurgery

\section{(d) Freedom from Symptomatic Radiation Necrosis (by Lesion)}

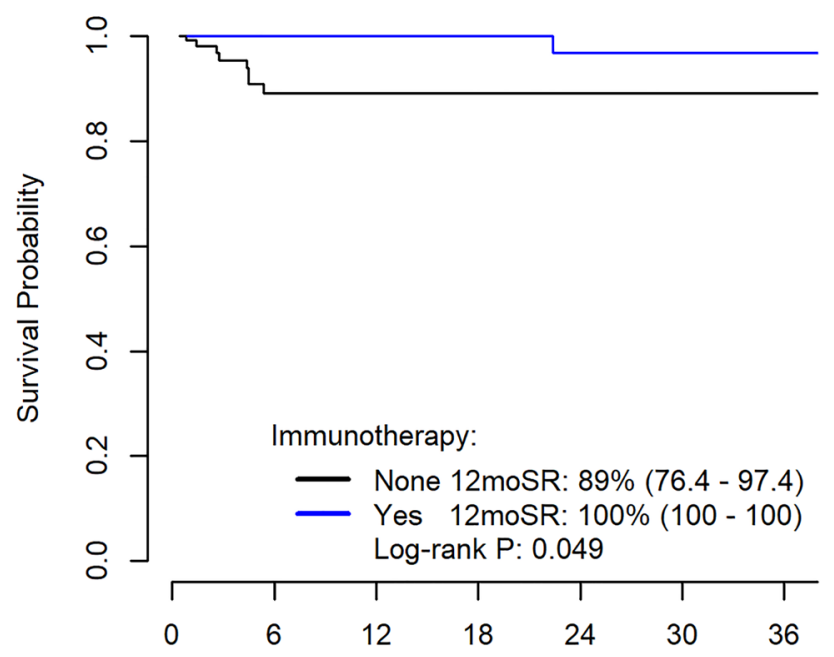

Months from completion of radiosurgery

comparing freedom from LF for subgroups stratified by IO receipt. d Kaplan-Meier curve comparing freedom from symptomatic RN for subgroups stratified by IO receipt

PDLDm (HR 0.307, CI 0.098-0.441) was the only significant predictor identified by the LASSO selector, with IO receipt not meeting statistical significance after inclusion of PDLDm (IO HR 0.363, CI 0.108-1.224, Table 3). Interaction effect testing demonstrated a significant interaction between IO receipt with SRS, peripheral dose per lesion size and freedom from local failure in our study cohort $(p=0.048)$. The hazard ratio for PDLDm with regards to 
Table 3 Multivariable Cox Frailty Models

\begin{tabular}{llccc}
\hline Predictor & Hazard Rate & LCI & UCI & p-value \\
\hline $\begin{array}{l}\text { Outcome: Overall Survival (by Patient)* } \\
\quad \text { IO }\end{array}$ & 0.482 & 0.265 & 0.879 & $\mathbf{0 . 0 1 7}$ \\
$\quad$ Outcome: Distant Brain Failure (by Patient)* & & & & \\
$\quad$ IO & 0.776 & 0.405 & 1.489 & 0.446 \\
Outcome: Local Failure (by Lesion)** & & & & \\
$\quad$ IO & 0.363 & 0.108 & 1.224 & 0.100 \\
PDLDm & 0.307 & 0.098 & 0.441 & $<.001$ \\
Outcome: Symptomatic Radiation Necrosis (by Lesion)*** & 0.101 & 0.010 & 0.988 & $\mathbf{0 . 0 4 9}$ \\
$\quad$ IO
\end{tabular}

IO immunotherapy, LCIlower confidence interval endpoint, UCIupper confidence interval endpoint, PDLDm peripheral dose per lesion diameter (Gy/mm on log-scale)

*LASSO model considered and excluded sex, age, Karnofsky Performance Scale, number of brain metastasis at diagnosis, at least one body metastasis at diagnosis, resection, recursive partitioning analysis, BRAF gene mutation, prior chemotherapy, prior immunotherapy, post immunotherapy, extracranial control

**LASSO model considered and excluded prior immunotherapy, tumor diameter (log-scale), mean dose, BRAF gene mutation

***LASSO model considered and excluded PDLDm, prior immunotherapy, tumor diameter (log-scale), mean dose, BRAF gene mutation freedom from LF significantly differed in this model for lesion treated with SRS alone (HR 0.117, CI 0.044-0.316) compared to lesions treated with SRS and IO (HR 0.634, CI $0.168-2.399$ ). Regarding symptomatic RN, IO receipt (HR 0.101 , CI $0.010-0.988$ ) was associated with significantly decreased risk of symptomatic RN with the log of tumor diameter, mean dose, PDLDm, prior chemotherapy, prior immunotherapy and BRAF mutational status excluded from the Cox frailty model.

\section{Lesion control}

The estimated 12-month $\mathrm{LC}$ rates for a $7.5 \mathrm{~mm}$ lesion receiving a peripheral SRS dose of $18 \mathrm{~Gy}(\mathrm{PDLDm}=2.4 \mathrm{~Gy} / \mathrm{mm})$ with versus without IO were $87.8 \%$ (95\% CI 79.0-98.3\%) versus $79.8 \%$ (95\% CI 55.1-93.8\%), respectively (Table 4).
As seen in Fig. 2, the greatest difference in 12-month LC occurs for the largest lesions (i.e. smallest values of PDLDm). For instance, the predicted 12-month lesion control for larger diameter lesions $(18 \mathrm{~mm})$ treated with SRS to a dose of $18 \mathrm{~Gy}$ with IO is predicted to be substantially higher $(82.6 \%$, CI $57.4-97 \%)$ relative to those treated with SRS alone $(25.0 \%$, CI $1.6-56.8 \%, \mathrm{p}<0.01$, Table 4$)$.

\section{Discussion}

In this retrospective investigation of a prospectively maintained cohort of consecutively treated MBM patients receiving SRS with or without IO, SRS with IO was associated with improved OS. SRS with IO was also associated with improved freedom from LF at 12 months by univariable

Table 4 Actuarial Estimates of 12-month lesion control stratified by immunotherapy receipt and peripheral dose per lesion diameter (18 Gy single fraction dose)

\begin{tabular}{|c|c|c|c|c|c|c|c|}
\hline \multirow{3}{*}{$\begin{array}{l}\text { Peripheral Dose /Lesion } \\
\text { Diameter (PDLDm) } \\
0.5 \mathrm{~Gy} / \mathrm{mm}\end{array}$} & \multirow{3}{*}{$\begin{array}{l}\text { Corresponding diameter }(\mathrm{mm}) \\
\text { for } 18 \text { Gy Dose }(\mathrm{mm})\end{array}$} & \multicolumn{3}{|l|}{ SRS Alone } & \multicolumn{3}{|c|}{ SRS with Immunotherapy } \\
\hline & & \multirow{2}{*}{$\begin{array}{l}12 \mathrm{mo} \mathrm{LC} \%(\%) \\
0.3\end{array}$} & \multicolumn{2}{|c|}{$\begin{array}{l}\text { Confidence Interval } \\
(\%)\end{array}$} & \multirow{2}{*}{$\begin{array}{l}12 \mathrm{mo} \mathrm{LC} \%(\%) \\
77.1\end{array}$} & \multicolumn{2}{|c|}{$\begin{array}{l}\text { Confidence } \\
\text { Interval (\%) }\end{array}$} \\
\hline & & & 0.0 & 15.4 & & 32.1 & 97.6 \\
\hline $1 \mathrm{~Gy} / \mathrm{mm}$ & 18 & 25.0 & 1.6 & 56.8 & 82.6 & 57.4 & 97.0 \\
\hline $2 \mathrm{~Gy} / \mathrm{mm}$ & 9 & 71.9 & 42.7 & 90.1 & 86.9 & 68.2 & 97.9 \\
\hline $2.4 \mathrm{~Gy} / \mathrm{mm}$ & 7.5 & 79.8 & 55.1 & 93.8 & 87.8 & 69.0 & 98.3 \\
\hline $5 \mathrm{~Gy} / \mathrm{mm}$ & 3.6 & 95.2 & 84.5 & 99.3 & 91.1 & 65.9 & 99.4 \\
\hline $10 \mathrm{~Gy} / \mathrm{mm}$ & 1.8 & 98.8 & 94.5 & 99.9 & 93.3 & 57.9 & 99.8 \\
\hline
\end{tabular}

$12 m o 12$ month, LC Local control, PDLDm parameter peripheral dose per lesion diameter 
12mo Local Control Probability by Dose/Diam

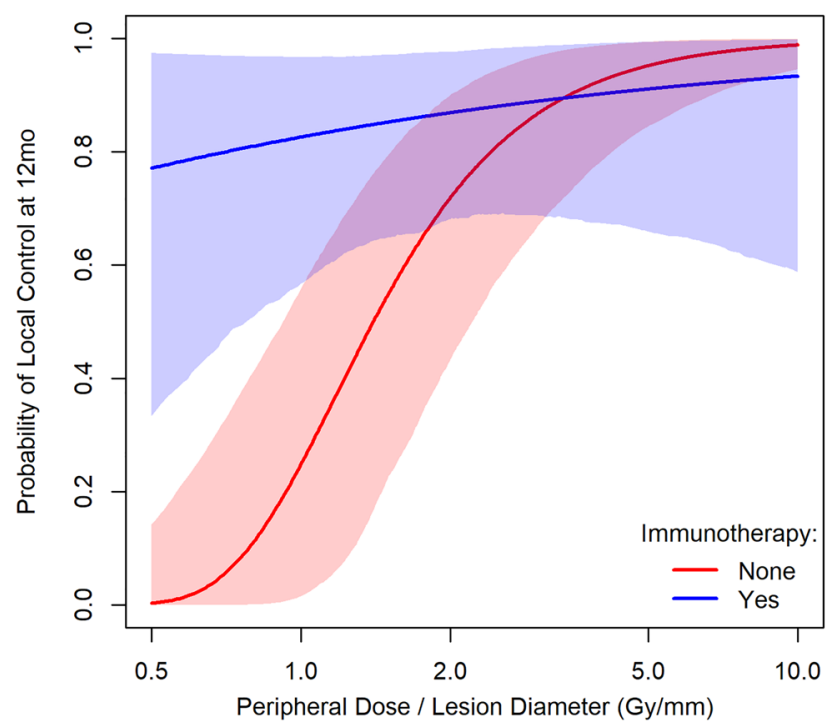

Fig. 2 Dose-Size Response (Local Control) for MBMs in cohort stratified by IO receipt

modeling with a trend towards improved freedom from LF when accounting for PDLDm. The SRS planning parameter PDLDm was selected as a significant predictor of LF. With regards to the primary study endpoint of determining whether a differential peripheral dose per lesion size relationship was present between MBM receiving SRS with versus without $\mathrm{IO}$, interaction effect testing demonstrated a significant interaction between peripheral dose per lesion size, IO receipt, and freedom from LF. SRS without IO was associated with poor lesion control for larger lesions (i.e. the smallest PDLDm values), whereas SRS with IO was associated with effective 12-month freedom from LF across all lesion sizes. In aggregate, our results suggest a potential difference in the effect of dose per lesion size by IO receipt for small MBMs treated with single-fraction SRS. Small MBMs treated with SRS and IO did not show the same LC benefit with escalated PDLDm as those treated with SRS alone, raising the question of whether peripheral doses of $>18 \mathrm{~Gy}$ are necessary to obtain high LC rates small MBMs treated with SRS and IO. This finding may be clinically significant, given the association between higher prescribed SRS doses and higher tissue V12 associated with risk of RN [14, 15].

Multiple prior investigations have associated excellent clinical outcomes including high rates of lesion control for MBM patients treated with SRS and IO compared to SRS alone [2, 3, 16, 17]. However, select series have reported relatively high rates of RN with $\mathrm{SRS}$ and IO [5, 6]-raising concern for potential increased risk of toxicity with combined modality treatment. The single fraction SRS dose recommendations provided by RTOG 90-05 [7] were designed with respect to maximal tolerated dose in lieu of optimal lesion control. This led to interest in better defining the single-fraction SRS dosing associated with optimal BM control [8, 9]. Further, the initial single fraction SRS dose level of $18 \mathrm{~Gy}$ for $\mathrm{BM} \leq 20 \mathrm{~mm}$ in maximum diameter in RTOG 90-05 demonstrated an 8\% risk of $\geq$ grade 3 chronic CNS toxicity. Consequently, there is interest in determining if de-escalated single fraction SRS doses with IO receipt could maintain excellent rates of $\mathrm{LC}$ with more favorable toxicity profiles [10].

Efforts to define SRS planning parameters balancing optimal brain metastatic control with minimizing normal tissue toxicity have been described in the available literature $[8,9,18,19]$. In a previous investigation performed at our center of 121 patients with 316 brain metastases from various primary tumor histologies, we previously found SRS dose per lesion size ( $\mathrm{Gy} / \mathrm{mm})$ to be significantly associated with lesion control for small $(\leq 3 \mathrm{~cm})$ brain metastases treated with SRS alone [8]. The current investigation similarly found that peripheral dose per lesion size (Gy/ $\mathrm{mm}$ ) was significantly associated with LC by multivariable modeling. Of interest, we noted a differential effect of dose per lesion size with regards to lesion control by IO receipt. Estimates of LC at 12 months remained relatively high for even large diameter lesions $(\geq 18 \mathrm{~mm})$ treated with SRS and IO. In contrast, a steep decline in actuarial LC rate at 12 months was present for larger diameter lesions treated with SRS alone. Rates of symptomatic RN were favorable with combined SRS and IO treatment.

Our institutional experience regarding optimal radiation dose per lesion size [8] was concordant with results published by Vogelbaum et al. [20] and Schoeggl et al. [21], which supported larger BM requiring higher RT doses using single-fraction SRS to achieve similar local control rates seen with SRS for smaller BM. RTOG 90-05 associated a maximum tolerated dose of $18 \mathrm{~Gy}$ for single fraction SRS for brain metastases $2.1-3.0 \mathrm{~cm}$, which was not an optimal dose for local control in our experience for lesions of this size [22]. There is concern for increased risk of RN with single-fraction dose escalation, particularly increased risk of symptomatic RN with single fraction SRS and concomitant immunotherapy [5]. Published literature supports consideration of hypofractionated RT for lesions $>2 \mathrm{~cm}$ [23-25], with favorable toxicity and efficacy profiles. The optimal dose of RT with regards to immunogenicity is unknown. However, emerging preclinical data has associated highly ablative single doses of RT ( $\geq 12-18 \mathrm{~Gy}$ ) to be associated with induction of DNA exonuclease Trex 1 - which may attenuate immunogeneicty by degrading cytosolic DNA [26]. Other high-volume centers have differing practices regarding criteria for hypofractionation or the use of staged SRS. Thus, we emphasize 
careful consideration of our cohort and treatment when extrapolating results to patients treated with different treatment schema.

There is a growing large body of literature on the possible mechanisms for enhanced efficacy of radiotherapy with the addition of immunotherapy. Some have suggested that the ablative doses of RT have immunostimulatory effects on the tumor microenvironment [27-29], including upregulation of cytotoxic CD8(+) T-cells, increased antigen presentation, upregulation of dendritic cells/other antigen presenting cells, which may increase the efficacy of IO. The optimal timing remains unclear, but we defer to concurrent treatment as defined by Qian et al. to mean within 4 weeks of initiating SRS [2].

These findings are hypothesis-generating and require further validation. Multiple limitations in addition to those inherent to retrospective analysis, such as selection bias, are present in this study and merit further discussion. Despite including 67 patients with over 240 MBM lesions, our patient and lesion sample size is relatively small. Despite SRS principles being strictly adhered to, it is possible that some level of confounding bias may be possible in a small cohort when using two different external beam radiation machines to deliver the single fraction dose. Moreover, there is a potential confounding effect of chemotherapy, the extent to which is unknown. SRS patients tended to have earlier treatment dates on average compared to the combined SRS and IO group and were thus likely to be pretreated with chemotherapy and other interventions prior to the introduction of immunotherapy in our institution. Additionally, MBMs $>2 \mathrm{~cm}$ are typically treated with fractionated stereotactic radiotherapy at our institution. Subsequently, the median lesion size for our cohort was relatively small at $<1 \mathrm{~cm}$, and the results of this study may not be applicable to larger MBM. Additionally, most lesions treated with either SRS alone or SRS with IO received $18 \mathrm{~Gy}$, and future studies utilizing differing single-fraction SRS doses are needed to provide additional nuance regarding the impact of SRS dose variance in the dose-size response relationship. Moreover, at the time of the study design, no reliable data was available to observe local control in a cohort receiving immunotherapy alone. Our institutional practice has been to refer melanoma patients with known brain metastases for radiation, and thus records for known brain metastases who did not receive radiation is not well documented. Practice patterns evolved at our institution over the study interval concordant with the advent of novel immune-modulating agents and subsequent literature demonstrating efficacy of these agents for patients with melanoma. We, for example, were not able to assess differences in outcomes as it related to type and dose of immunotherapy, which we believe should be a point for future investigation. While this is an acknowledged limitation, this study provides a hypothesis/ rationale for single fraction de-escalation with concomitant IO. Consequently, the median year of diagnosis was earlier for patients receiving SRS alone compared to those treated with SRS and IO. Lesions treated with SRS alone were also more heavily pre-treated with pre-SRS chemotherapy relative to those treated with SRS and IO, contributing to heterogeneity within these subgroups and potential modulation of RN. Lesions treated with SRS and IO were associated with favorable rates of symptomatic RN in our cohort. However, we do not propose combined SRS and IO approaches to be associated with decreased risk of $\mathrm{RN}$ relative to SRS alone.

\section{Conclusions}

The findings of this investigation support PDLDm as a useful SRS planning parameter associated with LC for small MBMs (median between cohorts were $<8 \mathrm{~mm}$ ). We identified a differential effect of dose per lesion size and LC by IO receipt with high rates of $L C$ at 12 months for even larger diameter lesions receiving SRS up to a dose of $18 \mathrm{~Gy}$ with IO. Therefore, peripheral doses $>18$ Gy may be unnecessary to obtain high LC rates for small MBMs treated with SRS and IO. This could support rationales for both planned and ongoing studies investigating SRS dose de-escalation in the setting of concomitant IO administration. Future prospective studies of larger cohorts treated with variable SRS doses are needed to validate our analyses.

Author contribution Study Conception: MY; Study Design: MY, JG. Data Collection: MY, JG. Statistical Analysis: JG. Interpretation of Results: MY, AR, JG, MS, DD, SW, EB, SFM, MEM. Draft Manuscript Preparation: MY, AR, ALO. All authors reviewed the result, critically read/revised the manuscript, and approved the final version of the manuscript.

Funding None.

Data availability The datasets generated and analyzed during the current study are available from the corresponding author on reasonable request.

\section{Declarations}

Conflict of interest None.

Ethical approval This article does not contain any studies with human participants or animals performed by any of the authors. 


\section{References}

1. Davies MA, Liu P, McIntyre S et al (2011) Prognostic factors for survival in melanoma patients with brain metastases. Cancer 117(8):1687-1696

2. Qian JM, Yu JB, Kluger HM, Chiang VL (2016) Timing and type of immune checkpoint therapy affect the early radiographic response of melanoma brain metastases to stereotactic radiosurgery. Cancer 122(19):3051-3058

3. Yusuf MB, Amsbaugh MJ, Burton E, Chesney J, Woo S (2017) Peri-SRS administration of immune checkpoint therapy for melanoma metastatic to the brain: investigating efficacy and the effects of relative treatment timing on lesion response. World Neurosurg 100:632-640.e634

4. Patel KR, Shoukat S, Oliver DE et al (2015) Ipilimumab and Stereotactic Radiosurgery Versus Stereotactic Radiosurgery Alone for Newly Diagnosed Melanoma Brain Metastases. Am J Clin Oncol 40(5):444-450

5. Martin AM, Cagney DN, Catalano PJ et al (2018) Immunotherapy and symptomatic radiation necrosis in patients with brain metastases treated with stereotactic radiation. JAMA Oncol 4(8):1123-1124

6. Du Four S, Janssen Y, Michotte A et al (2018) Focal radiation necrosis of the brain in patients with melanoma brain metastases treated with pembrolizumab. Cancer Med 7(10):4870-4879

7. Shaw E, Scott C, Souhami L et al (2000) Single dose radiosurgical treatment of recurrent previously irradiated primary brain tumors and brain metastases: final report of RTOG protocol 90-05. Int J Radiat Oncol Biol Phys 47(2):291-298

8. Amsbaugh MPJ, Yusuf MB, Dragun AE, Dunlap N, Guan T, Boling W, Rai S, Woo S (2016) Dose-volume response relationship for brain metastases treated with frameless single-fraction linear accelerator-based stereotactic radiosurgery. Cureus 8(4):587

9. Amsbaugh MJ, Yusuf MB, Gaskins J et al (2017) A dose-volume response model for brain metastases treated with frameless singlefraction robotic radiosurgery: seeking to better predict response to treatment. Technol Cancer Res Treat 16(3):344-351

10. McClelland S 3rd, Lautenschlaeger T, Zang Y et al (2020) Radiosurgery dose reduction for brain metastases on immunotherapy (RADREMI): A prospective phase I study protocol. Rep Pract Oncol Radiother 25(4):500-506

11. Ma L, Sahgal A, Larson DA et al (2014) Impact of millimeterlevel margins on peripheral normal brain sparing for gamma knife radiosurgery. Int J Radiat Oncol Biol Phys 89(1):206-213

12. Lin NU, Lee EQ, Aoyama $\mathrm{H}$ et al (2015) Response assessment criteria for brain metastases: proposal from the RANO group. Lancet Oncol 16(6):e270-278

13. Klein JP (2002) Survival analysis methods in cancer studies. Cancer Treat Res 113:37-57

14. Blonigen BJ, Steinmetz RD, Levin L, Lamba MA, Warnick RE, Breneman JC (2010) Irradiated volume as a predictor of brain radionecrosis after linear accelerator stereotactic radiosurgery. Int J Radiat Oncol Biol Phys 77(4):996-1001

15. Milano MT, Grimm J, Niemierko A et al (2020) Single- and multifraction stereotactic radiosurgery dose/volume tolerances of the brain. Int J Radiat Oncol Biol Phys. https://doi.org/10.1016/j. ijrobp.2020.08.013

16. Chen L, Douglass J, Kleinberg L et al (2018) Concurrent immune checkpoint inhibitors and stereotactic radiosurgery for brain metastases in non-small cell lung cancer, melanoma, and renal cell carcinoma. Int J Radiat Oncol Biol Phys 100(4):916-925
17. Lehrer EJ, Peterson J, Brown PD et al (2019) Treatment of brain metastases with stereotactic radiosurgery and immune checkpoint inhibitors: An international meta-analysis of individual patient data. Radiother Oncol 130:104-112

18. Dimitriadis A, Paddick I (2018) A novel index for assessing treatment plan quality in stereotactic radiosurgery. J Neurosurg 129(Suppl1):118-124

19. Flickinger JC, Lunsford LD, Kondziolka D (1992) Dose prescription and dose-volume effects in radiosurgery. Neurosurg Clin $\mathrm{N}$ Am 3(1):51-59

20. Vogelbaum MA, Angelov L, Lee SY, Li L, Barnett GH, Suh JH (2006) Local control of brain metastases by stereotactic radiosurgery in relation to dose to the tumor margin. J Neurosurg 104(6):907-912

21. Schoeggl A, Kitz K, Ertl A, Reddy M, Bavinzski G, Schneider B (1999) Prognostic factor analysis for multiple brain metastases after gamma knife radiosurgery: results in 97 patients. J Neurooncol 42(2):169-175

22. Shaw E, Scott C, Souhami L, Dinapoli R, Kline R, Loeffler J, Farnan N (2000) Single dose radiosurgical treatment of recurrent previously irradiated primary brain tumors and brain metastases: final report of RTOG protocol 90-05. Int J Radiat Oncol Biol Phys 47(2):291-298

23. Minniti G, Scaringi C, Paolini S, Lanzetta G, Romano A, Cicone F, Osti M, Enrici RM, Esposito V (2016) Single-fraction versus multifraction $(3 \times 9$ Gy) stereotactic radiosurgery for large $(>2$ $\mathrm{cm}$ ) brain metastases: a comparative analysis of local control and risk of radiation-induced brain necrosis. Int J Radiat Oncol Biol Phys 95(4):1142-1148

24. Minniti G, D’Angelillo RM, Scaringi C, Trodella LE, Clarke E, Matteucci P, Osti MF, Ramella S, Enrici RM, Trodella L (2014) Fractionated stereotactic radiosurgery for patients with brain metastases. J Neurooncol 117(2):295-301

25. Remick JS, Kowalski E, Khairnar R, Sun K, Morse E, Cherng HR, Poirier Y, Lamichhane N, Becker SJ, Chen S, Patel AN, Kwok Y, Nichols E, Mohindra P, Woodworth GF, Regine WF, Mishra MV (2020) A multi-center analysis of single-fraction versus hypofractionated stereotactic radiosurgery for the treatment of brain metastasis. Radiat Oncol 15(1):128

26. Vanpouille-Box C, Alard A, Aryankalayil MJ, Sarfraz Y, Diamond JM, Schneider RJ, Inghirami G, Coleman CN, Formenti SC, Demaria S (2017) DNA exonuclease Trex1 regulates radiotherapy-induced tumour immunogenicity. Nat Commun 8:15618

27. Donlon NE, Power R, Hayes C, Reynolds JV, Lysaght J (2021) Radiotherapy, immunotherapy, and the tumour microenvironment: turning an immunosuppressive milieu into a therapeutic opportunity. Cancer Lett 502:84-96

28. Colton M, Cheadle EJ, Honeychurch J, Illidge TM (2020) Reprogramming the tumour microenvironment by radiotherapy: implications for radiotherapy and immunotherapy combinations. Radiat Oncol 15(1):254-264

29. Zietman A, Yom S [Editors] (2020) Radiation therapy and the immune response. Int J Radiat Oncol Biol Phys 108(1):1-330

Publisher's Note Springer Nature remains neutral with regard to jurisdictional claims in published maps and institutional affiliations. 